\title{
The semantics and pragmatics of directional numeral modifiers *
}

\author{
Dominique Blok \\ Utrecht University
}

\begin{abstract}
Directional prepositions that can be used as numeral modifiers (directional numeral modifiers or DNMs) are different from other numeral modifiers that set an upper bound: they set a non-cancellable lower bound, their upper bound is cancellable, they are incompatible with the numeral at the bottom of the scale they quantify over, they are not downward monotone and do not license NPIs, and they interact differently with evaluative adverbs. This paper argues that the cause of all these differences is that DNMs assert a lower bound and implicate an upper bound, and that all class B numeral modifiers require quantification over a range of values. Crosslinguistic data support these claims.
\end{abstract}

Keywords: modified numerals, implicatures, ignorance effects, monotonicity, bottom-ofthe-scale effect, inquisitive semantics

\section{Introduction}

Directional numeral modifiers are expressions that can be used both as directional prepositions and as numeral modifiers. Expressions that have these two functions exist in a wide variety of languages, two of which are given in (1-2). ${ }^{1}$

\section{(1) English: up to}

a. Mary walked (all the way) up to the counter.

b. You can fit up to four suitcases in this car.

* I thank Rick Nouwen, Yaron McNabb, Stavroula Alexandropoulou, Elizabeth Coppock, Benjamin Spector, Lisa Bylinina, Hanna de Vries, Jeremy Kuhn, Annemarie van Dooren, the audience at the Proportions and Quantities workshop at DGfS 2015, and the SALT 25 reviewers and audience for helpful comments and feedback. I thank my informants for their judgments. The research leading to these results has received funding from the European Research Council under the European Union's Seventh Framework Programme (FP/2007-2013) / ERC Grant Agreement no. 313502.

1 More specifically, they exist in (at least) fifteen different languages: Danish, Dutch, English, Farsi/Persian, French, German, Greek, Hebrew, Hungarian, Italian, Polish, Romanian, Russian, Spanish, and Turkish. I obtained data on these languages by sending out questionnaires and interviewing informants. For all data and judgments I refer the reader to the complete data set, which can be found at http://dominiqueblok.org/work/.

(C)2015 Blok 
Blok

\section{(2) Turkish: kadar}

a. Golun sonuna kadar yuruduk. Lake edge KADAR we.walked.

'We walked up to the edge of the lake.'

b. 10 buvala kadar ucretsiz goturebiilirsiniz. 10 luggage KADAR for.free you.can.take.

'You can take up to ten items of luggage for free.'

Nouwen (2010) proposes a categorisation of numeral modifiers into two classes on the basis of whether or not they obligatorily give rise to ignorance effects. Schwarz, Buccola \& Hamilton (2012) point out that the English expression up to differs from the other members in its class and should be categorised separately.

The aim of this paper is twofold. The first objective is to show that not just up to but all directional numeral modifiers (henceforth: DNMs) crosslinguistically form a separate class in the domain of numeral modifiers that has properties that other numeral modifiers don't have. While other upper-bounded numeral modifiers such as at most set a strong upper bound, the upper bound of DNMs is defeasible. Furthermore, the lower bound set by DNMs, unlike their upper bound, is strong: DNMs entail the existence of their complement. Expressions such as at most lack a lower bound.

Moreover, DNMs display the so-called bottom-of-the-scale effect (Schwarz et al. note this about up to): they cannot refer to the lowest element of the scale they quantify over. Finally, their monotonicity properties differ from those of other upperbounded numeral modifiers such as at most. Whilst non-directional upper-bounded numeral modifiers are straightforwardly downward monotone and license NPIs, the monotonicity properties of directional numeral modifiers are more complex.

The second goal of the present work is to show that an implicature-based account can explain these properties of DNMs as well as generalise Schwarz et al.'s observation of the bottom-of-the-scale effect to all numeral modifiers. Following Coppock \& Brochhagen (2013), I implement this account in the framework of inquisitive semantics (e.g. Ciardelli, Groenendijk \& Roelofsen 2012).

In the following section, I will discuss the two central observations on which I base my analysis: the data concerning the strength of the lower bound of DNMs and the data that illustrates the defeasibility of their upper bound. In Section 3 I provide an analysis to account for these data. Section 4 contains a discussion of data relating to the bottom-of-the-scale effect, monotonicity, and evaluative adverbs. In Section 5 I implement the account in the framework of inquisitive semantics. Section 6 concludes. 
The semantics and pragmatics of directional numeral modifiers

\section{It's all in the bounds}

While there is a rich literature on modified numerals (e.g. Hackl 2000; Geurts \& Nouwen 2007; Büring 2008; Nouwen 2010; Cohen \& Krifka 2010, 2014; Coppock \& Brochhagen 2013; Kennedy 2015), no attention has been paid thus far to the bounds these expressions set. In this section I show that this is an area where directional numeral modifiers differ fundamentally from their non-directional counterparts.

\subsection{Lower bound}

A first difference between DNMs and other upper-bounded numeral modifiers is that DNMs entail the existence of a lower bound. ${ }^{2}$ Consider (3).

(3) a. At most three students will show up to the lecture, if any.

b. ? Up to three students will show up to the lecture, if any.

The cause of the infelicity of (3b) is the fact that the second part of the utterance, if any, contradicts the first part. As up to three students will show up entails that at least one student will show up, subsequently denying this claim leads to an incoherent utterance. (3a) is felicitous because at most three students show up is true in a world in which no students show up. In this sentence, the use of if any therefore does not lead to a contradiction.

(4) and (5) provide further evidence for this claim. ${ }^{3}$

(4) a. ? I expect to see at most ten people, but maybe no-one will show up.

b. I expect to see up to ten people, but maybe no-one will show up.

a. ? You're allowed to choose at most two presents, but you can also choose not to select any.

b. You're allowed to choose up to two presents, but you can also choose not to select any.

The infelicity of the a-sentences is a result of the use of but in these utterances. But indicates a contrast, but as at most ten/two is compatible with zero, there is no contrast to be found in these examples. Up to's incompatibility with zero causes an opposition in the b-sentences, licensing the use of but.

This observation holds for DNMs crosslinguistically. (6) illustrates the asserted lower bound of DNMs in Greek.

2 Throughout this paper I point out contrasts between DNMs and other numeral modifiers that set an upper bound. These include at most, maximally, and fewer/less than. For reasons of space, I use only at most in the examples.

3 These examples were provided by a reviewer of Console 2015. 
(6) a. Tha ine to poli 5 mathites sto seminario, an erthi (dhiladhi)

Will be at most 5 students to the seminar if comes (that is)

kanis.

anyone.

'There will be at most five students at the seminar, if any.'

b. \# Tha ine mehri 5 mathites sto seminario, an erthi (dhiladhi)

Will be MEHRI 5 students to the seminar if comes (that is)

kanis.

anyone.

'There will be up to five students at the seminar, if any.'

\subsection{Upper bound}

A second contrast between DNMs and non-directional upper-bounded numeral modifiers is that the upper bound set by DNMs appears to be weaker than the upper bound of expressions such as at most and less/fewer than. More specifically, the upper bound of DNMs can be cancelled. This is illustrated in (7) and (8).

(7) a. \# At most ten people died in the crash, perhaps even more.

b. Up to ten people died in the crash, perhaps even more.

a. \# Leftovers keep in the refrigerator for at most one week or more.

b. Leftovers keep in the refrigerator for up to one week or more. ${ }^{4}$

While the a-sentences clearly express a contradiction, the b-sentences are felicitous. As (9-10) exemplifies for Russian, this property holds for DNMs crosslinguistically. Here the judgments on the b-sentences reflect whether they are good continuations of the a-sentences.

(9) a. Do tridcati ljudej prišlo na večerinku.

Do thirty people came to party.

'Up to thirty people showed up at the party.'

b. Na samom dele, ja dumaju čto tam bylo 32 čeloveka.

In fact, I think that there were 32 people.

'In fact, I believe there were 32 people there.'

(10) a. Ne bolee tridcati ljudej prišlo na večerinku.

At most thirty people came to party.

'At most thirty people showed up at the party.'

4 Source: http://minimalistbaker.com/best-ever-5-minute-microwave-hummus/, last consulted 03-112014 
The semantics and pragmatics of directional numeral modifiers

b. ? Na samom dele, ja dumaju čto tam bylo 32 čeloveka.

In fact, I think that there were 32 people.

'In fact, I believe there were 32 people there.'

Furthermore, the upper bound of DNMs can be reinforced, unlike the upper bound of other upper-bounded numeral modifiers. (11) and (12) demonstrate this.

(11) a. \# You're allowed to choose at most ten presents, but no more than that.

b. You're allowed to choose up to ten presents, but no more than that.

a. \# Speeds of at most $120 \mathrm{~km} / \mathrm{h}$ are allowed, but you're not allowed to drive faster than that.

b. Speeds of up to $120 \mathrm{~km} / \mathrm{h}$ are allowed, but you're not allowed to drive faster than that.

This section shows that DNMs form a separate group in the domain of numeral modifiers in that they crosslinguistically differ from other numeral modifiers in a systematic way. In language after language, it can be observed that DNMs have a cancellable upper bound and a non-cancellable lower bound. As far as I know, no other upper-bounded numeral modifiers display these characteristics. In what follows, I will provide an account that explains these data and the data to be presented in Section 4.

\section{An implicature-based account}

\subsection{Two generalisations}

I claim that the differences between DNMs and other numeral modifiers are all effects of the following two generalisations: 1) The lower bound of DNMs is asserted while their upper bound is implicated; and 2) All so-called class B numeral modifiers (Nouwen 2010) require quantification over a range of values. In what follows, I will elaborate on these generalisations and explain how they can account for the data discussed in the previous sections.

The first generalisation follows straightforwardly from the observations about the bounds of DNMs: as DNMs are incompatible with the number zero, the existence of at least one element for which the relevant predicate holds must be entailed. Defeasibility and reinforceability are well-known characteristics of implicatures (Grice 1975). Given that the upper bound of DNMs has both of these properties, the conclusion that it is an implicature seems inescapable. 
Moreover, this generalisation ties in nicely with the meaning DNMs have in directional and temporal contexts. ${ }^{5}$ Here, too, there is a contextually determined 'lower bound' or starting point and a defeasible 'upper bound' or end-point. This can be observed in (13) and (14).

(13) Joan was at work (from 9am) until 10pm today. She may have even stayed later than that.

(14) Harry ran (from school) all the way up to his house. I think he may even have gone on to run to the football field after that.

In these cases, the relevant starting point is always there. It may be explicitly mentioned or left implicit, but it can never be nonexistent. The end-point, however, is defeasible; (13) and (14) show that it can be surpassed.

The second generalisation is inspired by Schwarz et al. (2012), who claim that the meaning of up to entails a range requirement. To see why I argue that not just up to or DNMs but all class B numeral modifiers have this requirement, let us take a brief detour to Nouwen's (2010) account of modified numerals.

\subsection{Ranges and ignorance}

Nouwen makes a distinction between two kinds of numeral modifiers: class A and class B modifiers. Class B modifiers are defined by their inability to refer to precise numbers. Class A modifiers, on the other hand, do have this ability.

As they cannot refer to specific cardinalities, class B numeral modifiers must always quantify over a plurality of elements or a range of admissible or possible options. This is shown in (15).

(15) a. Computers of this kind have at most 2GB of memory.

b. John is allowed to bring at most 10 friends.

In (15a), at most quantifies over a range of memory capacities of different computers, provided by the plural computers. In $(15 \mathrm{~b})$, the range is supplied by the modal allowed. The elements of the range are permissible numbers of friends John brings.

When a sentence has no operator that creates a range, such as a plural, a modal, or a universal quantifier, the modified numeral is interpreted with respect to a range of epistemic possibilities considered by the speaker: the ignorance effect. This is illustrated in (16).

(16) Stuart was absent for at most two weeks.

5 DNMs generally also have a temporal meaning. The fact that English uses until rather than up to in temporal contexts appears to be an idiosyncrasy of English. 
The semantics and pragmatics of directional numeral modifiers

The only interpretation of (16) is one where the speaker is unsure how long Stuart's absence lasted; she only knows that it was no more than two weeks. (16) excludes precise knowledge of the duration of absence, unlike its equivalent with a class $\mathrm{A}$ numeral modifier, given in (17).

(17) Stuart was absent for less than two weeks.

As demonstrated in (18), it is possible to specify the exact number referred to by a sentence with the class A modifier less than, but not with the class B modifier at most. This shows again that there is a clear incompatibility between class B numeral modifiers and precise numbers.

(18) a. Stuart was absent for less than two weeks; ten days to be precise.

b. \# Stuart was absent for at most two weeks; ten days to be precise.

Thus, numeral modifiers can be categorised according to their ability to refer to a specific cardinality. All languages I studied are sensitive to the A/B distinction: they all have expressions of both kinds.

\subsection{Ranges and DNMs}

As noted by both Nouwen and Schwarz et al., up to is a class B numeral modifier. (19) illustrates this.

\# There will be up to 100 people at the party, 90 to be precise.

Up to cannot refer to an exact number. In the absence of a modal, plural, or universal quantifier, this leads to ignorance effects. When an operator provides a range for $u p$ to to quantify over, as in (20), the most prominent reading is one where there is no ignorance. These facts are exactly the same as those we saw in the previous section. ${ }^{6}$

(20) a. Computers of this kind have up to $2 \mathrm{~GB}$ of memory.

b. John is allowed to bring up to 10 friends.

It is not only up to but rather all DNMs that belong in class B. An example from Romanian illustrates this fact in (21). The a-sentence and b-sentence are included to

6 In fact, there appears to be a difference between the prominence of ignorance effects of up to and at most. The sentences in (15) also have an ignorance reading. Under this reading, (15a) conveys that all computers have a certain amount of memory capacity and the speaker does not know the exact amount but she knows that it is no higher than 2GB. (15b) can express that the speaker is unsure about the amount of friends John is allowed to invite, but knows that this amount is lower than 11 . These readings seem to be less prominent if not absent for the sentences with up to in (20). I will leave this issue for further research. 
show that the language is sensitive to the class A/class B distinction. Hence, the fact that DNMs are incompatible with a precise number in Romanian can be attributed to the general classification discussed in the previous section and is not an idiosyncrasy of the lexical item până la.

(21) a. Un triunghi are mai puţin de 11 feţe.

A triangle has fewer than 11 sides.

b. \# Un triunghi are cel mult 10 feţe.

A triangle has at most 10 sides.

c. \# Un triunghi are până la 10 feţe.

A triangle has PÂNĂ LA 10 sides.

'A triangle has up to 10 sides.'

These facts show that it is not just up to, as Schwarz et al. claim, but all class B numeral modifiers that need a range of values to quantify over. This is the rationale behind the second generalisation I presented at the beginning of this section.

In summary, the two generalisations I make explain both the differences between the bounds of DNMs and those of other upper-bounded numeral modifiers and the ignorance effects all class B modifiers display. In the next section, I present three more pieces of data that can be accounted for by these generalisations.

\section{Additional data}

\subsection{The bottom-of-the-scale effect}

The first observation I will discuss here concerns the bottom-of-the-scale effect. Schwarz et al. (2012) note that up to, unlike at most, cannot be combined with the bottom element of the scale it quantifies over. (22) and (23) demonstrate this. ${ }^{7}$

(22) a. At most ten people died in the crash.

b. At most one person died in the crash.

(23) a. Up to ten people died in the crash.

b. \# Up to one person died in the crash.

They argue that it is not the number one but really the element at the bottom of the scale that is relevant by showing that, as the granularity of the scale changes, so does the bottom-of-the-scale element up to is incompatible with. If we assume that eggs are sold in cartons of six only, up to, but not at most, is incompatible with the number six, as (24) and (25) illustrate.

7 The English examples in this section are all from Schwarz et al. (2012). 
The semantics and pragmatics of directional numeral modifiers

(24) a. He bought at most a dozen eggs.

b. He bought at most half a dozen eggs.

(25) a. He bought up to a dozen eggs.

b. \# He bought up to half a dozen eggs.

(24-25) exemplify a case where the bottom-of-the-scale element is higher than one. Because the only possible amounts of eggs that can be bought in this context are numbers divisible by six, the relevant scale is $[6,12,18,24, \ldots]$ rather than $[1,2$, $3,4, \ldots]$. When this number is lower than one, the bottom-of-the-scale effect still manifests itself, as can be observed in (26-27), where judgments are based on the assumption that cakes are sold per slice. Assuming that there are 12 slices in a cake, the scale that is relevant in this context is $\left[\frac{1}{12}, \frac{1}{6}, \frac{3}{12}, \frac{1}{3}, \ldots\right]$.

(26) a. She bought at most one cake.

b. She bought at most one slice of cake.

(27) a. She bought up to one cake.

b. \# She bought up to one slice of cake.

As these examples show, up to is perfectly compatible with the number one when this number is not the bottom-of-the-scale element.

The bottom-of-the-scale effect doesn't only hold for the English expression up to but is in fact a property of all DNMs I studied. My informants were consistent in confirming that DNMs are incompatible with the lowest number on the relevant scale in their language. An example in Farsi is given in (28).

a. Hadde aksar yek nafar dar tasadof mord.

At most one person in.the crash died.

'At most one person died in the crash.'

b. \# Ta yek nafar dar tasadof mord.

TA one person in.the crash died.

'Up to one person died in the crash.'

If we accept the two generalisations presented in the previous section, this phenomenon is easily explained. Let us make the natural assumption that the relevant scale in (22-23) consists of the set of whole numbers. Then at most in (22b) quantifies over the scale $[0,1]$. At most does not set a lower bound and is thus compatible with zero. Up to in (23b), on the other hand, does set a lower bound. If we take this lower bound to be 1, the scale up to quantifies over is [1]. If we assume it is higher than 1 , the scale is empty. In either case, there is no range of numbers for up to to quantify over, so the range requirement is violated. 
Since all class B numeral modifiers are subject to the range requirement, I predict that the class B modifiers that set an upper bound all display the bottom-of-the-scale effect. As (29) demonstrates, this prediction is borne out.

\# At most 0 people died in the crash.

Here at most can only quantify over [0], which violates the range requirement, so the result is infelicitous.

It seems, then, that the range requirement and its interactions with the bounds of numeral modifiers not only explains the bottom-of-the-scale effect of DNMs but can also account for data on other class B modifiers.

\subsection{Monotonicity}

Another difference between DNMs and other upper-bounded numeral modifiers has to do with the monotonicity properties of these expressions. Schwarz et al. (2012) claim that up to is not downward monotone, although this is a property you would expect to see in a numeral modifier that sets an upper bound. The authors claim that while (30b) straightforwardly follows from (30a), this is not so for the pair in (31).
a. At most three students smoke. $=$
b. At most three students smoke cigars.
a. Up to three students smoke. $\not \models$
b. Up to three students smoke cigars.

The argument Schwarz et al. present for their claim that (31b) does not follow from (31a) is that in a situation where the speaker knows that one, two, or three students smoke and that one or two - but not three — students smoke cigars, (31a) is 'true and appropriate', but (31b) is not.

Two things should be teased apart here. Schwarz et al. argue that (31b) may fail to be true and appropriate in a situation where (31a) is both true and appropriate. It is important to recognise that truth and appropriateness are two very different notions that should not be conflated when judging entailments.

To illustrate, in the scenario described above, (30a) is also true and appropriate while (30b) is not. Claiming that the number of cigar smokers is 'at most three' when you know that this number is one or two but not three constitutes a clear quantity violation: why use the less informative 'at most three' when you could have used the more informative 'at most two'? The example provided by Schwarz et al. could therefore just as well have been used to argue that at most is not downward entailing.

A better argument to claim that up to is not downward monotone is the fact that it does not license NPIs, unlike at most. This is shown in (32-33). 
The semantics and pragmatics of directional numeral modifiers

(32) a. At most three people had ever been in this cave.

Krifka (2007)

b. At most three students give a damn about Pavarotti.

Chierchia \& McConnell-Ginet (2000)

(33) a. * Up to three people had ever been in this cave.

b. * Up to three students gave a damn about Pavarotti.

If we follow Ladusaw's (1979) theory of NPI-licensing, this suggests that up to is either non-monotone or upward entailing. Schwarz et al. propose a non-monotone semantics for up to. To see whether they are on the right track, it is necessary to take a closer look at the patterns in (30-31).

Although Schwarz et al. claim that the entailment pattern in (31) does not hold, my informants did not straightforwardly reject this pattern in their languages. Furthermore, they did not reject the opposite pattern, either (where the b-sentence entails the a-sentence), and were thus unable to determine the direction of entailment. This is in contrast with their judgments on (30), which corresponded to Schwarz et al.'s judgments. Furthermore, my informants' judgments were uniform in confirming that DNMs do not license NPIs, as (34) exemplifies for Dutch.

a. Er hoeven maximaal vijf studenten te komen. There must-NPI maximally five students to come.

'At most five students have to show up.'

b. * Er hoeven tot vijf studenten te komen. There must-NPI TOT five students to come. 'Up to five students have to show up.'

What can we make of the fact that my informants think that there is something to be said for both the entailment pattern in (31) and the opposite pattern? Again, it will prove to be valuable to tease apart the semantics and the pragmatics of the sentences. According to the present proposal, DNMs assert a lower bound and are thus upward entailing. The valid pattern is $(31 \mathrm{~b}) \models(31 \mathrm{a})$. However, the pragmatics of DNMs contributes an upper bound to the meaning. This means that there is a pragmatic force interfering with the judgments and leading informants to think that (31b) may follow from (31a). Because the semantics and pragmatics each nudge the informants in a different direction, determining which way the entailment goes becomes quite a challenge. This explains my informants' hesitation to reject either pattern. Furthermore, the fact that DNMs are predicted to be upward entailing is compatible with the fact that they crosslinguistically fail to license NPIs.

So far, I have shown that my account not only explains the defeasibility of the upper bound and indefeasibility of the lower bound of DNMs, but also accounts for the bottom-of-the-scale effect and entailment patterns. In what follows, I will 
present one final piece of data that can be explained by the two generalistaions I propose.

\subsection{Evaluative adverbs}

A final argument for the analysis proposed here is that evaluative adverbs do not target the upper bound of DNMs while they do target the upper bound of other numeral modifiers that set an upper bound. To see why this is relevant, consider the sentences in (35).

a. Fortunately, some students attended the wedding.

Nouwen (2006)

b. Fortunately, the soup is warm.

It is standardly assumed that the prejacents of these sentences assert respectively that at least some students attended the wedding and that the soup is at least warm. They implicate that not all students attended the wedding and that the soup is not hot. As remarked by Nouwen (2006), fortunately targets only the assertion of these sentences, not the implicature. That is, (35a) cannot be taken to mean that the speaker is happy that not all students attended the wedding. (35b) conveys that the speaker is glad that the soup is at least warm; he is not necessarily glad that the soup is not hot.

Nouwen uses this observation to explain the difference between almost and not quite. Both almost and not quite have two meaning components: a proximal component and a negative component. As (36) illustrates, fortunately targets the proximal component in the case of almost and the negative component in the case of not quite.

(36) a. Fortunately, almost all my friends will attend my wedding.

b. Fortunately, not quite all my friends will attend my wedding.

(36a) expresses that the speaker is happy that the number of friends that will show up to his wedding is close to his total number of friends; fortunately targets the proximal component of almost. In (36b), on the other hand, the speaker conveys that he is pleased that some friends will not attend the wedding; fortunately targets the negative component of not quite.

A similar contrast can be observed if one compares up to to at most. Consider the sentences in (37).

(37) a. Fortunately, up to 100 people will attend my wedding.

b. Fortunately, at most 100 people attended my wedding. 
The semantics and pragmatics of directional numeral modifiers

While the speaker of (37a) expresses her joy about the high number of guests that will attend the wedding, the person uttering (37b) conveys that she is happy that no more than 100 people will be there. ${ }^{8}$ In parallel to almost and not quite, fortunately targets the positive component of up to and the negative component of at most.

This contrast between DNMs and other upper-bounded numeral modifiers holds crosslinguistically. All of my informants agreed that evaluative adverbs like fortunately target the positive component of DNMs and the negative component of at most in their languages. (38) and (39) provide an illustration of this fact for Italian.

(38) a. Fortunatamente, posso prendere fino a cinque giorni di ferie.

Fortunately, I.can take FINO A five days of time.off.

'Fortunately, I can get up to five days off.'

b. ? Fortunatamente, quel pessimo cantante canterà fino a cinque

Fortunately, that bad singer will.sing FINO A five

canzoni.

songs.

'Fortunately, that bad singer will sing up to five songs.'

a. ? Fortunatamente, posso prendere al massimo cinque giorni di ferie.

Fortunately, I.can take at most five days of time.off.

'Fortunately, I can get at most five days off.'

b. Fortunatamente, quel pessimo cantante canterà at most cinque

Fortunately, that bad singer will.sing at most five canzoni.

songs.

I asked my informants to judge these sentences under the assumption that getting time off work is pleasant while having to listen to the performance of a bad singer is not. The discrepancies in sentences like (38b) and (39a) confirm the intuitions presented above.

These facts can only be explained if one assumes that the upper bound of DNMs is not part of their asserted content. Of course these data do not provide conclusive evidence that the upper bound is a conversational implicature; it could be a conventional implicature or a presupposition. However, they are compatible with an analysis that treats the upper bound of DNMs as an implicature, with the cancellability of the upper bound providing more evidence.

8 In fact, DNMs seem to also have a proximal component in some cases. I will leave this issue for future research. 


\subsection{Interim conclusion}

I have shown that assuming that DNMs assert their lower bound and implicate their upper bound and that all class B numeral modifiers are subject to a range requirement can explain more than just the defeasibility facts in Section 2 and the ignorance effects of class B quantifiers. It can account for the bottom-of-the-scale effect that not only DNMs but all class B modifiers display, it can make sense of the difference in monotonicity properties between DNMs and other upper-bounded numeral modifiers, and it can explain why evaluative adverbs fail to target the upper bound of DNMs while they do target the upper bound of other numeral modifiers that set an upper bound. The next section contains the technical implementation of this theory in inquisitive semantics.

\section{Implementation in inquisitive semantics}

\subsection{The framework}

The main difference between inquisitive semantics (e.g. Ciardelli, Groenendijk \& Roelofsen 2009; 2012) and classical semantics is that the framework of inquisitive semantics comprises a richer notion of meaning than classical semantics. Rather than merely expressing truth-conditional content, the meaning of a proposition in inquisitive semantics is taken to be a proposal to update the common ground. This can be done by uttering a proposition that consists of a single possibility or by uttering one that comprises multiple possibilities. Possibilities are sets of worlds (or classical propositions), which means propositions are sets of sets of worlds. When a single possibility is expressed, the meaning of the proposition is equivalent to its meaning in classical semantics. In Coppock \& Brochhagen (2013), a proposition that comprises more than one possibility is referred to as an interactive possibility.

$$
\varphi \text { is interactive iff } \llbracket \varphi \rrbracket \text { contains more than one possibility }
$$

As will become clear in the following sections, the distinction between interactive and non-interactive propositions is a useful tool to account for the ignorance effects certain numeral modifiers give rise to as well as the semantics of DNMs. The version of inquisitive semantics I adopt here is unrestricted inquisitive semantics or Inq, in which possibilities can non-trivially contain other possibilities. In the next section, I show how Coppock and Brochhagen use this framework in their account of modified numerals before moving on the formalisation of my own account. 
The semantics and pragmatics of directional numeral modifiers

\subsection{Inquisitive semantics in Coppock and Brochhagen (2013)}

Coppock and Brochhagen propose a discourse-based account of the superlative modified numerals at least and at most, which is partly based on Coppock \& Beaver's $(2011 ; 2013)$ analysis of only. Although the authors deal with a wide range of data, I focus here on how they account for the ignorance effects of superlative and other class B modifiers. I also restrict myself to discussing their semantics of the upper-bounded at most. This semantics, slightly modified to a version that takes numerals and degree predicates as arguments, is given in (41), with $\mathrm{MAX}_{s}$ defined as in (42).

$$
\begin{aligned}
& \llbracket \text { at most } \rrbracket=\left\{\lambda n \lambda P . f\left\{p \cap \operatorname{MAX}_{s} P(n) \mid p \leq_{s} P(n)\right\} \mid f \text { is a choice function }\right\} \\
& \operatorname{MAX}_{s}(p)=\lambda w . \forall p^{\prime} \in \hat{s}: p^{\prime}(w) \rightarrow p \geq_{s} p^{\prime}
\end{aligned}
$$

The possibility $p$ such that no possibilities in the state $\hat{s}$ are stronger than $p$

Here $\hat{s}$ is the question under discussion relative to a state $s$. A state is a contextually determined partially ordered set of possibilities (i.e. a partially ordered set of sets of worlds) and the question under discussion is the set of possibilities that are ordered in the state - possibilities in the state that are unordered with respect to one another are not part of the question under discussion. $P$ is a degree predicate, $n$ is a degree, and $p$ and $p^{\prime}$ are possibilities.

At most, then, is defined as the set of possibilities, intersected with the strongest possibility in $P(n)$, that are as highly or less highly ordered in $s$ than $P(n)$. For example, (43a) has the denotation in (43b). Assuming a monotone semantics of bare numerals, $P(5)$ denotes the worlds $\left\{w_{5}, w_{6}, w_{7}, \ldots\right\}$, where $w_{n}$ stands for the world in which Claire drank exactly $n$ beers. ${ }^{9}$ From these worlds, MAX $_{s}$ removes the worlds that are contained in alternatives higher than $P(5)$, as illustrated in (43c), which results in an upper bound of five.

a. Claire drank at most five beers.

b. $\left\{p \cap \operatorname{MAX}_{s} P(5) \mid p \leq{ }_{s} P(5)\right\}$ where $P(5)$ stands for $\exists x[\# x=5 \wedge$ beer $(x) \wedge \operatorname{drank}(x)$ (Claire) $]$

$$
\begin{aligned}
\text { c. } & =\left\{p \cap\left\{w_{0}, \ldots, w_{5}\right\} \mid p \in\left\{p_{0}, \ldots, p_{5}\right\}\right\} \\
& =\left\{\left\{w_{0}, \ldots, w_{5}\right\},\left\{w_{1}, \ldots, w_{5}\right\},\left\{w_{2}, \ldots, w_{5}\right\},\left\{w_{3}, \ldots, w_{5}\right\},\left\{w_{4}, w_{5}\right\},\left\{w_{5}\right\}\right\}
\end{aligned}
$$

What is crucial about this analysis is that the denotation of at most is interactive: it contains more than one possibility. Coppock and Brochhagen propose that it is this feature that distinguishes it and other class B numeral modifiers from class A

9 This is a simplified model where for each $n$ there is only one world in which Claire drank exactly $n$ beers. 
numeral modifiers, which denote a singleton set. Coppock and Brochhagen posit that if a speaker utters an interactive proposition, she must not know which of the possibilities in the proposition are true. If she had known this, she would have uttered a non-interactive proposition with only one possibility. Coppock and Brochhagen call this the Maxim of Interactive Sincerity, which they define as in (44).

(44) If $\varphi$ is interactive, then $\varphi$ is interactive in the speaker's information set

To avoid violations of this maxim, a speaker uttering an interactive proposition must always have an interactive information state: the speaker must be entertaining multiple possibilities in her mind. Class A modifiers do not pose this restriction: since they result in non-interactive propositions, the speaker's information set can but does not have to contain a plurality of possibilities. This, according to the authors, accounts for the fact that class B expressions obligatorily come with ignorance effects in the absence of an operator that provides a range, whereas class A expressions are compatible with ignorance but do not require it.

In sum, Coppock and Brochhagen's account of ignorance effects hinges on the idea that class B numeral modifiers are always interactive, and that it is infelicitous to utter a proposition with multiple possibilities when you know which possibility is the true one. In what follows, I will show that the ignorance effects of DNMs as well as their other properties can also be accounted for in this framework.

\subsection{DNMs in inquisitive semantics}

Coppock and Brochhagen use a maximality operator in their semantics for at most to reflect the fact that it sets a semantic upper bound. For DNMs, which lack a semantic upper bound, I propose the semantics in (45).

$$
\llbracket \text { up to } \rrbracket=\left\{\lambda n_{d} \lambda P_{\langle d, t\rangle} \cdot f\{P(m) \mid s \leq m \leq n\} \mid f \text { is a choice function }\right\}
$$
where $s>0$ and $s \neq n$

This definition says that up to is the set of possibilities $P(m)$ - where $P$ is a degree predicate and $m$ is a numeral of type $d$ - such that $m$ is between a contextually determined lower bound $s$ and the number modified by up to, $n$. The range requirement is reflected in the requirement that the scale consist of more than one element $(s \neq n)$. The starting point of the scale, $s$, is determined by the context of the utterance. As illustrated in Section 4.1, this number can be lower or higher than one but not zero. To illustrate how this semantics works, let us consider (46).

Up to ten people died in the crash.

I assume with Hackl (2000) and Nouwen (2010) that a sentence with a modified numeral has the structure in (47): up to $\mathrm{n}$ is a generalised quantifier over degrees that 
The semantics and pragmatics of directional numeral modifiers

scopes over the rest of the sentence. It leaves a degree trace, which is an argument of the silent counting quantifier many, defined in (48). The semantics of (46) is then as in (49).

[up to $10[\lambda n[n$-many people died in the crash] ] ]

$$
\begin{aligned}
& \llbracket \text { many } \rrbracket=\lambda n_{d} \lambda P_{\langle e, t\rangle} \lambda Q_{\langle e, t\rangle} \cdot \exists x[\# x=n \wedge P(x) \wedge Q(x)] \\
& \{f\{\lambda w \exists x[\# x=m \wedge \text { people }(x)(w) \wedge \text { died-in-the-crash }(x)(w)] \mid \\
& s \leq m \leq 10\} \mid f \text { is a choice function }\} \\
& =\{\lambda w \exists x[\# x=m \wedge \text { people }(x)(w) \wedge \text { died-in-the-crash }(x)(w)] \mid s \leq m \leq 10\} \\
& =\left\{p_{1}, p_{2}, p_{3}, \ldots, p_{10}\right\} \\
& \text { where } p_{n}=\left\{w_{n}, w_{n+1}, w_{n+2}, \ldots \infty\right\}
\end{aligned}
$$

The informational content of (46) - the union of the set of possibilities - is now equivalent to that of the proposition At least one person died in the crash. However, the structure of the proposition will enable us to derive the upper-bound implicature. To do this, we can use Coppock and Brochhagen's inquisitive exhaustification procedure, defined in (50).

$\operatorname{EXH}(P, \hat{s})=\left\{p-q \mid p \in P \wedge q=\left\{w \mid \exists q^{\prime} \in \hat{s}\left[w \in q^{\prime} \wedge p \nsubseteq q^{\prime}\right]\right\}\right\}$ where $P$ is the proposition and $\hat{s}$ is the question under discussion

For each possibility $p$ in $P$, this removes the worlds in $q$, where $q$ is the set of worlds that are in a possibility $q^{\prime}$ in $\hat{s}$ that is not entailed by $p$. This leads to the outcome in (51) for (46).

$$
\begin{aligned}
& P=\left\{p_{1}, p_{2}, \ldots, p_{10}\right\}\left(=\left\{\left\{w_{1}, w_{2}, w_{3}, \ldots\right\},\left\{w_{2}, w_{3}, w_{4}, \ldots\right\}, \ldots,\left\{w_{10}, w_{11}, w_{12}, \ldots\right\}\right\}\right) \\
& \hat{s}=\left\{q_{0}^{\prime}, q_{1}^{\prime}, q_{2}^{\prime}, q_{3}^{\prime}, q_{4}^{\prime}, \ldots\right\}\left(=\left\{\left\{w_{0}, w_{1}, w_{2}, \ldots\right\},\left\{w_{1}, w_{2}, w_{3}, \ldots\right\}, \ldots\right\}\right) \\
& \operatorname{EXH}(P, \hat{s})=p_{1}-q=p_{1}-\left\{w_{2}, w_{3}, w_{4}, \ldots\right\}=\left\{w_{1}\right\} \\
& p_{2}-q=p_{2}-\left\{w_{3}, w_{4}, w_{5}, \ldots\right\}=\left\{w_{2}\right\} \\
& \quad \ldots \\
& p_{10}-q=p_{10}-\left\{w_{11}, w_{12}, w_{13}, \ldots\right\}=\left\{w_{10}\right\} \\
& =\left\{\left\{\boldsymbol{w}_{1}\right\},\left\{\boldsymbol{w}_{2}\right\}, \ldots,\left\{\boldsymbol{w}_{10}\right\}\right\}
\end{aligned}
$$

As (51) illustrates, the exhaustivity procedure removes all worlds above $w_{10}$ from the informational content, resulting in an implicated upper bound of 10. This is due to the interactive structure of (46). If the denotation of the sentence had been a singleton set (i.e. $\left\{p_{1}\right\}$ ), the application of EXH would have resulted in $\left\{\left\{w_{1}\right\}\right\} .{ }^{10}$ It is thus the complex inner structure of the proposition and not its informational content that enables us to derive the implicature.

10 This may be a good representation of bare numerals in the inquisitive framework. 
As can be seen above, statements with up to lead to interactive propositions, just like statements with other class B numeral modifiers. This means that ignorance effects come about in the same way: raising ten different possibilities, as the speaker of (46) does, is infelicitous when you know which possibility is the true one. In exactly the same way as it did in the previous section for at most, the Maxim of Interactive Sincerity accounts for the ignorance effects of DNMs.

There is one instance where this mechanism does not work, and it is in cases where the lowest number on the relevant scale is used. The proposition denoted by (52) is the singleton set $\left\{p_{1}\right\}$, or, after applying exhaustivity, $\left\{\left\{w_{1}\right\}\right\}$.

$$
\text { \# Up to one person died in the crash. }
$$

This sentence does not violate the Maxim of Interactive Sincerity, which only states that the information set of the speaker needs to be interactive if the proposition is interactive. Coppock and Brochhagen can therefore not account for the cases in (53).

a. \# At most 0 people died in the crash.

b. \# At most no-one showed up to the reception.

Again, it is clear that a range requirement is needed to rule out these sentences, both for DNMs and for other class B numeral modifiers that set an upper bound.

Although my analysis can also be formalised in the classical degree semantics framework used by Nouwen (2010) and Schwarz et al. (2012) (as is done in Blok 2015), I hope to have shown that by drawing a distinction between interactive and non-interactive propositions, the inquisitive framework provides a natural way to express the semantics of DNMs in terms of speaker possibilities.

\section{Conclusion}

I have shown that DNMs differ fundamentally from non-directional numeral modifiers that set an upper bound. I take the essential difference to be the fact that DNMs assert their lower bound and implicate their upper bound. I demonstrated that this difference along with the assumption that all class B modifiers are subject to a range requirement can account for lower and upper bound cancellation facts, the bottom-of-the-scale effect and interactions with evaluative adverbs, monotonicity, and negative polarity items.

More broadly, this study shows that numeral modifiers do not differ from each other solely with respect to whether or not they can refer to a specific cardinality, which is the difference the literature has focused on so far, but that the nature of the bounds they set also plays a fundamental role in determining their semantic properties. Crosslinguistic data support these claims. 
The semantics and pragmatics of directional numeral modifiers

\section{References}

Blok, Dominique. 2015. The semantics and pragmatics of directional numeral modifiers. Unpublished manuscript, available at: http://www.dominiqueblok. org/work.

Büring, Daniel. 2008. The least at least can do. In West Coast Conference on Formal Linguistics (WCCFL), vol. 26, 114-120.

Chierchia, Gennaro \& Sally McConnell-Ginet. 2000. Meaning and Grammar: An Introduction to Semantics. Cambridge, MA: MIT Press. http://dx.doi.org/10. $2307 / 415078$.

Ciardelli, Ivano, Jeroen Groenendijk \& Floris Roelofsen. 2009. Attention! Might in inquisitive semantics. In Satoshi Ito, Ed Cormany \& David Lutz (eds.), Semantics and Linguistic Theory (SALT), vol. 19, 91-108. http://dx.doi.org/10. 3765/salt.v0i0.2520.

Ciardelli, Ivano, Jeroen Groenendijk \& Floris Roelofsen. 2012. Inquisitive Semantics NASSLLI 2012 lecture notes.

Cohen, Ariel \& Manfred Krifka. 2010. Superlative quantifiers as modifiers of metaspeech acts. In Michael Glanzberg, Barbara Partee \& Jurgis Škilters (eds.), The Baltic International Yearbook of Cognition, Logic and Communication, vol. 6, 1-56. Kansas: New Prairie Press. http://dx.doi.org/10.4148/biyclc.v6i0.1578.

Cohen, Ariel \& Manfred Krifka. 2014. Superlative quantifiers and metaspeech acts. Linguistics \& Philosophy 37. 41-90. http://dx.doi.org/10.1007/ s10988-014-9144-x.

Coppock, Elizabeth \& David Beaver. 2011. Sole sisters. In Neil Ashton, Anca Chereches \& David Lutz (eds.), Semantics and Linguistic Theory (SALT), vol. 21, 197-217. http://dx.doi.org/10.3765/salt.v0i0.2615.

Coppock, Elizabeth \& David Beaver. 2013. Principles of the exclusive muddle. Journal of Semantics 31(3). 371-432. http://dx.doi.org/10.1093/jos/fft007.

Coppock, Elizabeth \& Thomas Brochhagen. 2013. Raising and resolving issues with scalar modifiers. Semantics \& Pragmatics 6(3). 1-57. http://dx.doi.org/10.3765/ sp.6.3.

Geurts, Bart \& Rick Nouwen. 2007. At least at al.: The semantics of scalar modifiers. Language 83(3). 533-559. http://dx.doi.org/10.1353/lan.2007.0115.

Grice, Paul. 1975. Logic and conversation. In P. Cole \& J. Morgan (eds.), Syntax and Semantics 3: Speech Acts, 41-58. New York: Academic Press.

Hackl, Martin. 2000. Comparative quantifiers. PhD Thesis, Department of Linguistics and Philosophy, Massachusetts Institute of Technology.

Kennedy, Chris. 2015. A 'de-Fregean' semantics (and neo-Gricean pragmatics) for modified and unmodified numerals. Semantics and Pragmatics 8(10). 1-44. http://dx.doi.org/10.3765/sp.8.10. 
Krifka, Manfred. 2007. More on the difference between more than two and at least three. http://amor.cms.hu-berlin.de/ h2816i3x/Talks/SantaCruz2007.pdf.

Ladusaw, William. 1979. Polarity sensitivity as inherent scope relations. $\mathrm{PhD}$ Thesis. University of Texas at Austin.

Nouwen, Rick. 2006. Remarks on the polar orientation of almost. In J. van de Weijer \& B. Los (eds.), Linguistics in the Netherlands, vol. 23, 162-173. Amsterdam: AvT Publications, John Benjamins Publishing Company. http://dx.doi.org/10. 1075/avt.23.17nou.

Nouwen, Rick. 2010. Two kinds of modified numerals. Semantics \& Pragmatics 3(3). 1-41. http://dx.doi.org/10.3765/sp.3.3.

Schwarz, Bernhard, Brian Buccola \& Michael Hamilton. 2012. Two types of class B numeral modifiers: A reply to Nouwen 2010. Semantics \& Pragmatics 5(1). 1-25. http://dx.doi.org/10.3765/sp.5.1.

Dominique Blok

Trans 10

3512 JK Utrecht

Netherlands

D.Blok@uu.nl 